\title{
Evidence for the presence of a vasoactive substance (possibly involved in the aetiology of cerebral arterial spasm) in cerebrospinal fluid from patients with subarachnoid haemorrhage
}

\author{
D. J. BOULLIN 1 , J. MOHAN², AND D. G. GRAHAME-SMITH \\ MRC Unit of Clinical Pharmacology, and Department of Neurological Surgery, \\ Radcliffe Infirmary, Oxford
}

SYNOPSIS Cerebrospinal fluid from patients with subarachnoid haemorrhage and cerebral arterial spasm contracted the isolated basilar artery and potentiated contractions produced by 5-hydroxytryptamine, noradrenaline, and six prostaglandins. The unidentified substance in cerebrospinal fluid probably plays a role in the aetiology of cerebral arterial spasm.

We have recently described the contractile responses of the isolated human basilar artery to noradrenaline (NA), 5-hydroxytryptamine (5-HT), and contractile substances derived from human blood (Starling et al., 1975). The purpose of these experiments was to develop a human cerebral arterial model which could be used as a basis for investigating a possible association between subarachnoid haemorrhage (SAH) and the presence of vasoactive substances in cerebrospinal fluid (CSF) which may be responsible for the prolonged arterial spasm that often accompanies subarachnoid haemorrhage.

We now report the results of experiments in which we have tested the responses of CSF taken from a number of patients admitted to the Radcliffe Infirmary, Oxford, for clipping of cerebral aneurysms after subarachnoid haemorrhage. We have evidence for the presence of a vasoactive substance, or substances, as yet unidentified, in the CSF of these patients with a predominance of this factor in those patients

\footnotetext{
${ }^{1}$ Address for correspondence: D. J. Boullin, MRC Unit and University Department of Clinical Pharmacology, Radcliffe Infirmary, Woodstock Road, Oxford OX2 6HE.

2 Present address: University Department of Neurosurgery, Manchester Royal Infirmary, Oxford Road, Manchester.

(Accepted 10 March 1976.)
}

showing angiographic evidence of preoperative spasm of the cerebral arteries.

Some preliminary data have recently been presented to the Medical Research Society (Boullin et al., 1975a) and at the 5th Congress of the European Association of Neurosurgical Societies (Boullin et al., 1975b).

\section{METHODS}

SUBJECTS Thirty-four patients presented with subarachnoid haemorrhage from ruptured aneurysms. They underwent bilateral carotid and/or vertebral angiography to determine the site of aneurysm and the presence or absence of cerebral arterial spasm. Lumbar CSF was obtained preoperatively from these patients two to 10 hours after angiography. The age, sex, and sites of aneurysm and spasm are given in Table 1. Control CSF was obtained from 12 patients (three females, nine males, aged 19-53 years) undergoing myelography for suspected prolapsed intervertebral disc.

ASSESSMENT OF CEREBRAL ARTERIAL SPASM The presence or absence of cerebral arterial spasm was assessed by comparison of the angiograms of the affected and unaffected sides. No attempt was made to quantitate the degree of spasm by magnification angiography. The patients were then divided into two groups: with or without spasm. 
TABLE 1

LOCATION OF SITES OF ANEURYSM AND CEREBRAL SPASM, TIME INTERVAL BETWEEN HAEMORRHAGE AND CSF COLLECTION, AND RESPONSE OF BASILAR ARTERY TO CSF IN PATIENTS WITH SUBARACHNOID HAEMORRHAGE

\begin{tabular}{|c|c|c|c|c|c|c|}
\hline No. & $\begin{array}{l}\text { Age } \\
(y r)\end{array}$ & Aneurysm site & Spasm site & $\begin{array}{l}\text { Subjects } \\
\text { (no.) }\end{array}$ & $\begin{array}{c}\text { Time interval between } \\
\text { haemorrhage and CSF } \\
\text { collection (days) }\end{array}$ & $\begin{array}{l}\text { Basilar artery } \\
\text { response to CSF }\end{array}$ \\
\hline \multicolumn{7}{|c|}{ Males with spasm } \\
\hline \multirow[t]{8}{*}{9} & $49-66$ & MCA & MCA & 2 & $0.3,9$ & $+t$ \\
\hline & & Ant. comm. A & $\mathrm{ACA}$ & 1 & 5 & + \\
\hline & & Ant. comm. A & diffuse & 1 & 5 & + \\
\hline & & Post. comm. A+MCA & $\mathbf{M C A}+\mathbf{A C A}$ & 1 & 10 & + \\
\hline & & $\mathbf{M C A}+\mathbf{A C A}$ & $\mathbf{M C A}+\mathbf{A C A}$ & 1 & 10 & + \\
\hline & & MCA & $\mathbf{M C A}+\mathbf{A C A}$ & 1 & 6 & $\mathbf{R}$ \\
\hline & & Post. comm. A & $\mathbf{I C A}+\mathbf{A C A}$ & 1 & 12 & NR \\
\hline & & $\mathrm{ACA}$ & $\mathrm{ACA}$ & 1 & 12 & NR \\
\hline \multicolumn{7}{|c|}{ Females with spasm } \\
\hline \multirow[t]{10}{*}{13} & $38-65$ & ICA & ICA & 1 & 13 & + \\
\hline & & Ant. comm. A & ACA & 2 & $4,7^{*}$ & $+t^{*}$ \\
\hline & & PICA & VB & 1 & 6 & + \\
\hline & & MCA & MCA & 1 & 5 & + \\
\hline & & Pericallosal & Pericallosal & 1 & 23 & + \\
\hline & & $\mathbf{A C A}+\mathbf{I C A}$ & diffuse & 1 & 8 & + \\
\hline & & Post. comm. A & $\mathbf{I C A}+\mathbf{A C A}$ & 1 & 6 & + \\
\hline & & Post. comm. A & MCA & 1 & 5 & + \\
\hline & & ICA & $\mathrm{ACA}$ & 3 & $6,4,9$ & $+*, R, N R$ \\
\hline & & Basilar & VB & 1 & 3 & NR \\
\hline \multicolumn{7}{|c|}{ Males with no spasm } \\
\hline \multirow[t]{4}{*}{6} & $30-51$ & Ant. comm. A & & 2 & 4,6 &,$+ N R$ \\
\hline & & Pericallosal + Ant. comm. A & & 1 & 7 & \\
\hline & & MCA & & 2 & 5,9 & NR, NR \\
\hline & & $\mathbf{A C A}+\mathbf{M C A}$ & & 1 & 0.3 & NR \\
\hline \multicolumn{7}{|c|}{ Females with no spasm } \\
\hline \multirow[t]{4}{*}{6} & $17-56$ & ICA & & 2 & $0.3,4$ & $+, \mathbf{R}, \mathbf{N}$ \\
\hline & & Ant. comm. A & & 1 & 8 & $\mathbf{R}$ \\
\hline & & MCA & & 2 & 4,13 & NR, NR \\
\hline & & ICA + Ant. comm. A & & $\hat{1}$ & 1 & NR \\
\hline
\end{tabular}

*=multiple bleeds. $\mathbf{M C A}=$ middle cerebral. Ant. comm. $\mathbf{A}=$ anterior communicating. Post. comm. A=posterior communicating. $\mathbf{A C A}=$ anterior cerebral. ICA = internal carotid. PICA = posterior inferior cerebellar. $\mathbf{M C A}=$ middle cerebral. VB=vertebrobasilar.

The greatest care was taken to exclude all patients with even doubtful spasm or traces of spasm from the no spasm group (NVS). On the other hand the much larger group with spasm (VS) included patients showing a range from only very slight spasm to very severe spasm as illustrated in Fig. 3.

COLLECTION AND PREPARATION OF CSF After collection the CSF was immediately cooled to $4^{\circ} \mathrm{C}$ and centrifuged at $3000 \mathrm{~g} / 5 \mathrm{~min}$ to remove cells and debris. The clear supernatant was then frozen at $-20^{\circ} \mathrm{C}$, lyophilized at $-80^{\circ} \mathrm{C}$, and then the dried material was stored at $-20^{\circ} \mathrm{C}$ until required.

ISOLATED BASILAR ARTERY The setting up of the isolated preparation has been described in detail in our earlier paper (Starling et al., 1975). The only modifications made subsequently were as follows:

1. Instead of preparing the muscle by making alternate transverse cuts, the artery was cut spirally.
This change did not influence the qualitative or quantitative responses of the arteries to drugs.

2 . We changed to an electrical system for recording alterations in arterial tension. The muscle was attached to an isotonic transducer (Model 1326, C. F. Palmer Ltd., Lane End Road, High Wycombe, Bucks.) and the changes in arterial tone were recorded on a Tekman TE200 single channel potentiometric recorder (Tekman Electronics Ltd., Bicester, Oxon.).

ARTERIAL RESPONSES TO CSF AND DRUGS The artery was maintained in $\mathrm{Krebs}$ solution at $37^{\circ} \mathrm{C}$ as described earlier (Starling et al., 1975). Drugs were applied to the artery in a $13 \mathrm{ml}$ organ bath in volumes not exceeding $200 \mu \mathrm{l}$ and normally in the range $20-100 \mu \mathrm{l}$. Addition of these volumes of Krebs solution did not change arterial tone.

Aliquots of lyophilized CSF were reconstituted in 0.1 volumes of distilled water and added to the 
organ bath in volumes of $50-500 \mu$. These volumes of normal CSF did not affect arterial tone (Starling et al., 1975).

In experiments where the effects of ions were investigated, $\mathrm{MgCl}_{2}, \mathrm{CaCl}_{2}$, or $\mathrm{KCl}$ were added to the organ bath to increase the molar concentrations of $\mathrm{Mg}^{++}, \mathrm{Ca}^{++}$, or $\mathrm{K}^{+}$as described in the Results section.

DRUGS 5-HT, noradrenaline, and atropine were obtained from Sigma Chemical Company, Kingston, Surrey. Methysergide and 2-bromolysergic acid diethylamide from Sandoz Ltd., Feltham, Middlesex; phentolamine from Ciba Laboratories Ltd., Horsham, West Sussex, and mepyramine from May \& Baker Ltd., Dagenham, Essex. The following prostaglandins were kindly donated by Dr J. E. Pike, The Upjohn Company, Kalamazoo, MI 49001, USA: $\mathrm{E}_{1}\left(\mathrm{PGE}_{1}\right) ; \mathrm{E}_{2}\left(\mathrm{PGE}_{2}\right) ; \mathrm{A}_{1}\left(\mathrm{PGA}_{1}\right) ; \mathrm{A}_{1}$ $\left(\mathrm{PGA}_{2}\right) ; \mathrm{F}_{1 \alpha} \quad\left(\mathrm{PGF}_{1 \alpha}\right) ; \mathrm{F}_{2 \alpha}$-tromethamine salt $\left(\mathrm{PGF}_{2 \alpha}\right)$.

All drugs were made up in distilled water and the concentrations are expressed in terms of molarities of the base and represent the concentration of drug present in the organ bath.

\section{RESULTS}

Table 1 demonstrates that 16 of 22 patients presented with arterial spasm. In our series of 34 patients 22 had some degree of spasm, whereas only 12 had no angiographic evidence for spasm. The subjects showed a wide age range from 17 to 66 years.

SITES OF ANEURYSM In both males and females most of the sites of aneurysm were at the usual locations on the circle of Willis. Multiple aneurysms were seen in four patients (three in males; one in a female). In males with arterial spasm six out of 10 aneurysm sites were located on the middle and anterior cerebral arteries and the remainder on the anterior and posterior communicating arteries.

In females with spasm, the situation was more variable: the distribution was wider involving six sites on the circle of Willis (Table 1).

In the smaller number of patients without spasm, the distribution of aneurysms followed that of the subjects with spasm.

SITES OF SPASM In both males and females, identifiable spasm was largely confined to the internal carotid, middle, and anterior cerebral arteries. These three arteries accounted for all the sites of spasm in the males and for nine out of 13 in the females, the remainder being on the vertebrobasilar system (two cases), the pericallosal artery (one case); diffuse unilateral spasm occurred in two cases, one male and one female.

CSF COLLECTION The time interval between haemorrhage and CSF collection was variable with a mean value of seven days for both male and female groups with spasm. In subjects without spasm the mean interval was shorter, only four days in both male and female groups.

Where the arterial responses to CSF are concerned, there appears to be no relation between the basilar artery response and the other parameters noted in Table 1.

ACTIVITY OF ISOLATED BASILAR ARTERIES As described earlier, many basilar arteries contract to drugs when tested up to five days after death (Starling et al., 1975). Having now obtained numerous arteries within 24 hours of death, we observe that some display spontaneous rhythmic contractions (Figs. 4 and 6). This rhythm is not abolished by application of CSF, but it can be masked by those drugs which cause the basilar artery to contract as described below.

RESPONSES TO DRUGS We have previously reported that the basilar artery contracts in response to 5-HT and NA (Starling et al., 1975). Subsequently, we have tested several prostaglandins on the basilar artery: $\mathrm{PGE}_{1}, \mathrm{PGE}_{2}, \mathrm{PGA}_{1}, \mathrm{PGA}_{2}$, $\mathrm{PGF}_{1 \alpha}$, and $\mathrm{PGF}_{2 \alpha}$ and found that all these compounds contracted the artery in concentrations $0.1 \mathrm{nmol} / \mathrm{ml}$. The time course of prostaglandin-induced contractions was similar to those produced by 5-HT or NA-that is, maximal responses were obtained within 10 to 15 minutes.

In our earlier paper (Starling et al., 1975) we reported that 5-HT- and NA-induced contractions were maximal in three to five minutes; however, we now find that some basilar arteries do have a longer time course of response to these amines of up to 15 minutes. Additionally, we have now constructed dose/response curves for 5-HT, $\mathrm{NA}$, and $\mathrm{PGE}_{2}$ using the cumulative dose procedure in order to overcome the problems of tachyphylaxis. Figure 1 shows cumulative dose/ 


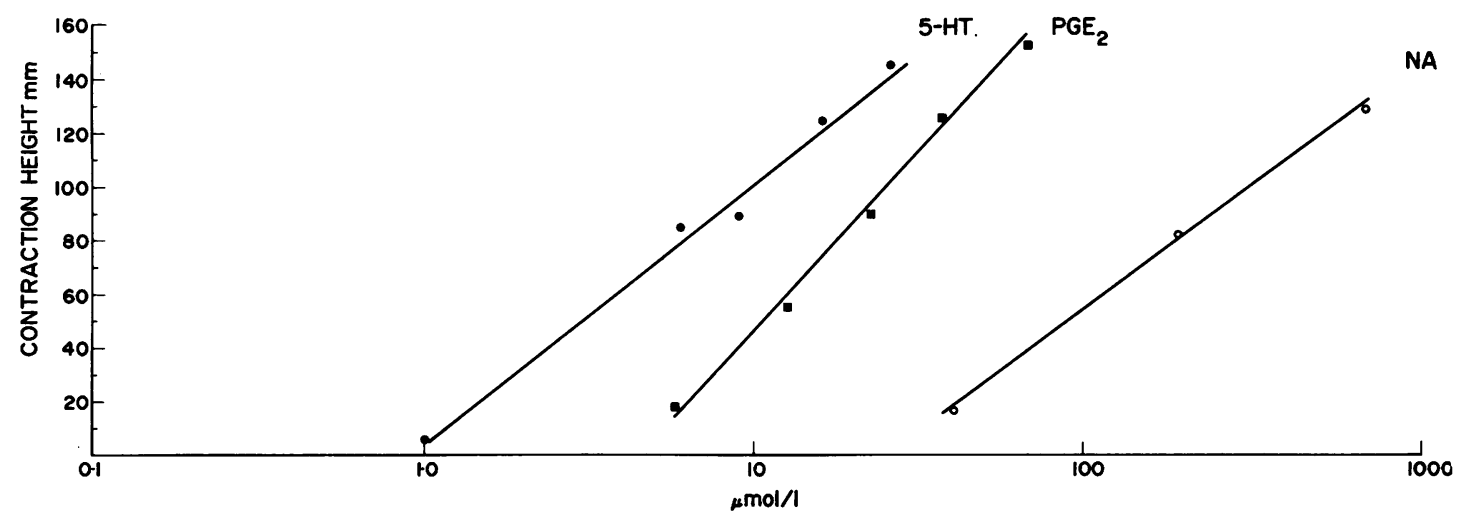

FIG. 1 Dose relationships for contraction of basilar arteries by 5-HT, noradrenaline, and prostaglandin $E_{2}$. Contraction height ( $\mathrm{mm}$, arithmetical scale, ordinate) is plotted against drug concentration in the isolated organ bath ( $\mu$ mol/l, logarithmic scale, abscissa). Each point represents the mean value obtained in three separate experiments, using cumulative concentrations of drug.

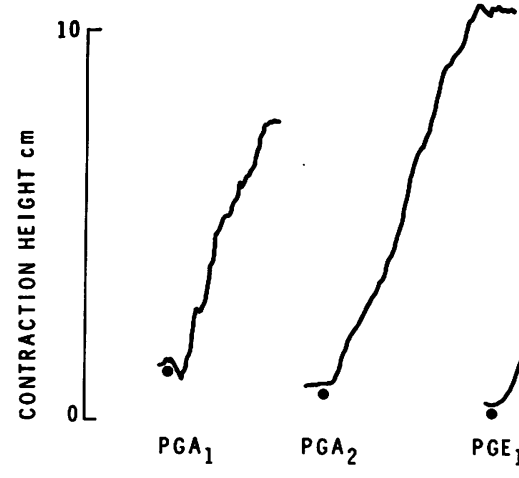

PG'S $0.2 \mu \mathrm{mol} / \mathrm{l}$

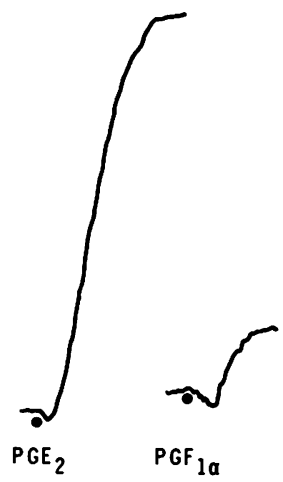

$P_{2} \quad P G F_{1 a}$

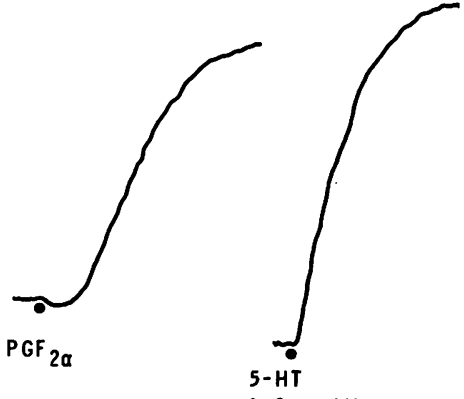

$1.0 \mu \mathrm{mol} / \mathrm{I}$

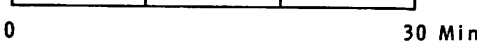

FIG. 2 Comparative responses of the basilar artery to six prostaglandins and 5-HT added at the filled circles, Contractions are plotted as contraction height ( $\mathrm{cm}$, ordinate) in relation to time (min, abscissa). The time scale refers only to the duration of the contractions. When the contractions were maximal the drugs were washed out of the isolated organ bath, and the preparation was rested until the arterial length had returned to the pre-drug value. All responses were obtained on the same artery and the total duration of the experiment was about four hours.

response curves for 5-HT, NA, and $\mathrm{PGE}_{2}$. It is clear that the pharmacological potencies of 5-HT and $\mathrm{PGE}_{2}$ are much greater than NA. This is in agreement with animal studies.

We have also studied the responses of the basilar artery to other prostaglandins than $\mathrm{PGE}_{2}$ although we have not prepared dose/response curves. Figure 2 shows the responses of a basilar artery to six different prostaglandins in comparison with 5-HT-induced contractions. All the 
prostaglandins that were tested contracted the artery showing similar potencies, except for $\mathrm{PGF}_{1 \alpha}$, which had less contracting activity.

RESPONSES TO CSF Table 2 shows that contractile substances were present in the CSF of 16 out of 22 subjects with vasospasm, but in only two out of 12 without spasm. The contractions were slowly developing, taking up to six hours to reach a maximum. These responses were not blocked by the 5-HT antagonist, 2-bromolysergic acid diethylamide (BOL), or the $a$-adrenergic blocker phentolamine in concentrations of $10 \mathrm{nmol} / \mathrm{ml}$, which Starling et al. (1975) found

TABLE 2

RESPONSES OF BASILAR ARTERIES TO CSF FROM PATIENTS WITH SUBARACHNOID HAEMORRHAGE WITH OR WITHOUT ARTERIAL SPASM

\begin{tabular}{|c|c|c|c|c|c|}
\hline \multirow[b]{2}{*}{ Sex } & \multirow[b]{2}{*}{ No. } & \multirow{2}{*}{$\begin{array}{c}\text { Arterial } \\
\text { spasm }\end{array}$} & \multicolumn{3}{|c|}{ Basilar artery response to CSF } \\
\hline & & & Contraction & Relaxation & No response \\
\hline $\mathbf{M}$ & 9 & Yes & 6 & 1 & 2 \\
\hline $\mathbf{F}$ & 13 & Yes & 10 & 1 & 2 \\
\hline $\mathbf{M}$ & 6 & No & 1 & 1 & 4 \\
\hline $\mathrm{F}$ & 6 & No & 1 & 2 & 3 \\
\hline
\end{tabular}

antagonized contractions produced by $5-\mathrm{HT}$ or NA. CSF-induced contractions were also unaffected by the antihistamine, mepvramine.

A typical response is shown in Fig. 3 and illustrates the consistent finding that the time course of these contractions was quite different from that seen with 5-HT, NA, or prostaglandins.

We tested each sample of CSF on at least three different arteries and found that the responses were qualitatively consistent. That is, those samples which produced contractions invariably did so, while those which did not produce any effect and those which relaxed also did so consistently.

It must be emphasized that the time course of the relaxations was quite different from the contractions. The former were of rapid onset, within 30 seconds, and were usually transient, lasting no longer than 15 minutes and never longer than 30 minutes, when the response returned to the baseline. In some instances, the initial transient relaxations were followed by the slowly developing sustained contractions described above (Fig. 4).

Control CSF from 12 patients undergoing myelography did not affect arterial responses. This agrees with our earlier experiments using CSF from patients undergoing surgery for reasons

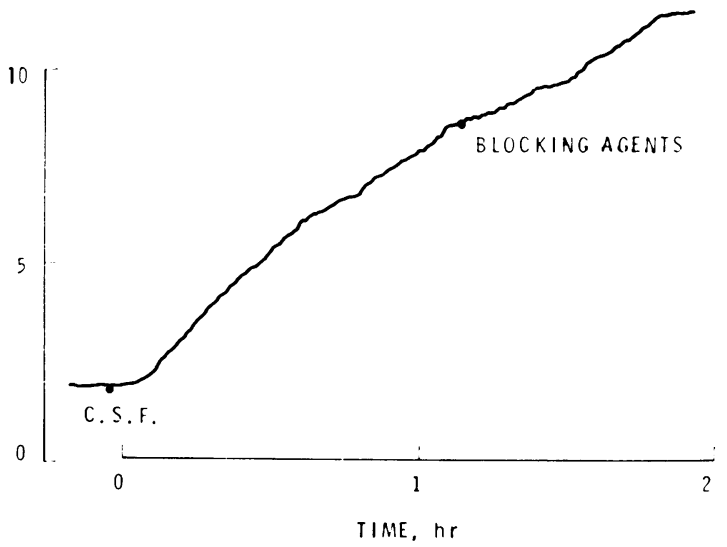

FIG. 3 Vertebral angiogram showing an aneurysm on the vertebral artery and intense spasm, and the contractile response of basilar artery to CSF taken, within four hours of angiography. Lyophilized CSF (200 $\mu l)$ reconstituted in distilled water (see Methods section) was added at the filled circle on the right record. Approximately 60 min later $10 \mu \mathrm{mol} / \mathrm{l}$ 2-bromolysergic acid diethylamide, phentolamine, and mepyramine were added together at the filled circle labelled 'blocking agents'. The patient was a 42 year old female. 

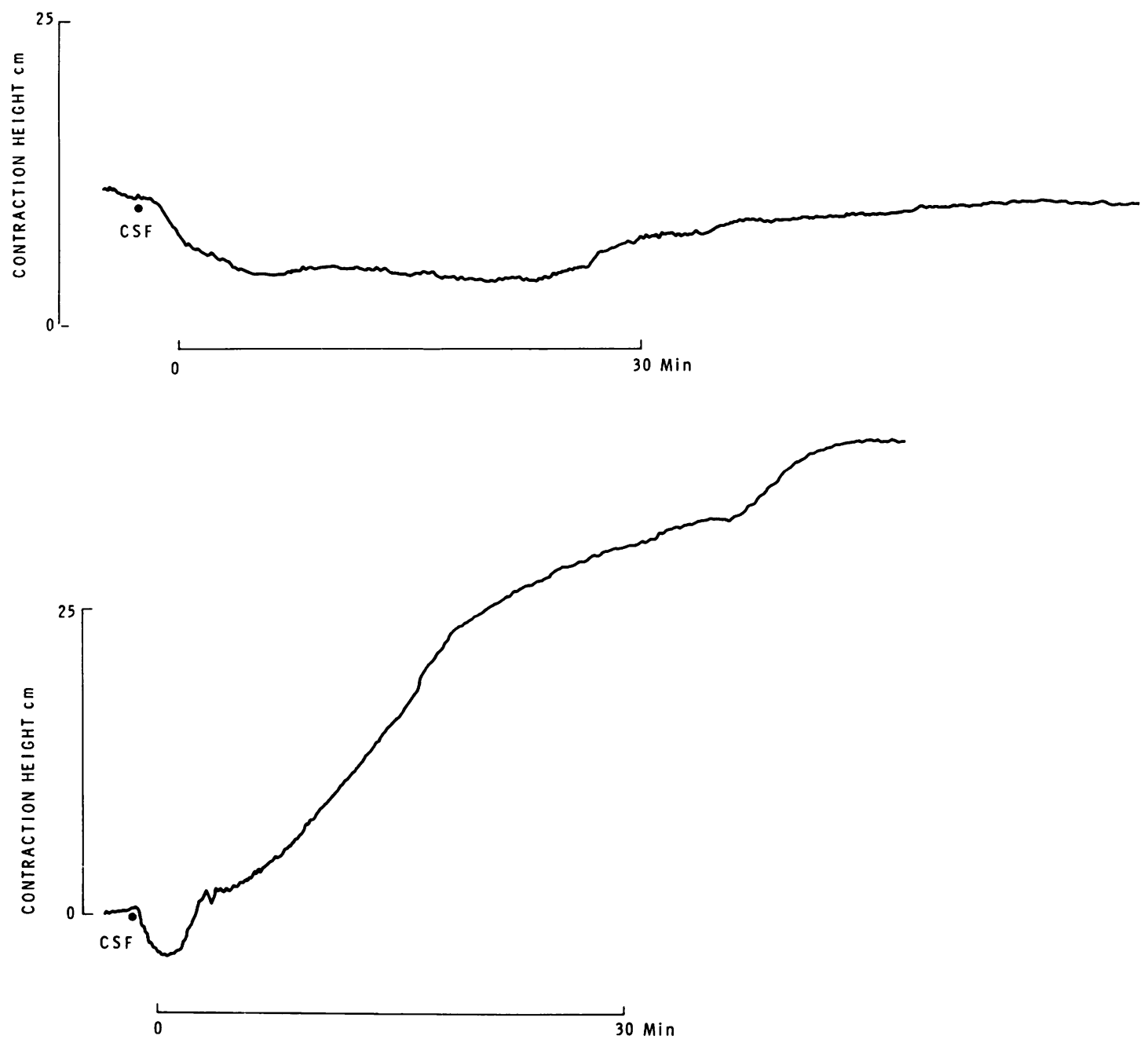

FIG. 4 Relaxations of basilar arteries produced by CSF from patients with subarachnoid haemorrhage and arterial spasm. Contractions recorded as described in Fig. 3. Upper record: relaxation of artery produced by $200 \mu$ l lyophilized CSF added at the filled circle, in a 44 year old male. Lower record: basilar artery response to CSF from another patient. Note that the transient relaxation is followed in 5 min by a slowly developing sustained contraction.

other than subarachnoid haemorrhage (Starling et al., 1975).

EFFECTS OF CATIONS ON THE BASILAR ARTERY The question arose as to whether the contractile responses were due to changes in the ionic composition of the CSF. Accordingly, we investigated the effects of $\mathrm{Mg}^{++}\left(\mathrm{MgSO}_{4} 7 \mathrm{H}_{2} \mathrm{O}\right.$ or $\left.\mathrm{MgCl}_{2}\right)$, $\mathrm{Ca}^{++}\left(\mathrm{CaCl}_{2}\right)$, and $\mathrm{K}^{+}(\mathrm{KCl})$ on the basilar artery. Figure 5 compares the dose/response relationships for changes in arterial length obtained with increasing concentrations of these ions. $\mathrm{K}^{+}$induced concentration-dependent contractions which were much smaller than those produced by CSF, prostaglandins, 5-HT, or NA even at the highest concentration tested $(100 \mathrm{mmol} / \mathrm{l})$. Furthermore, both $\mathrm{Ca}^{++}$and $\mathrm{Mg}^{++}$relaxed the artery in a concentration-dependent manner but $\mathrm{Ca}^{++}$acted in extremely high concentrations (greater than $10 \mathrm{mmol} / \mathrm{l})$ and $\mathrm{Mg}^{++}$-induced relaxations were 

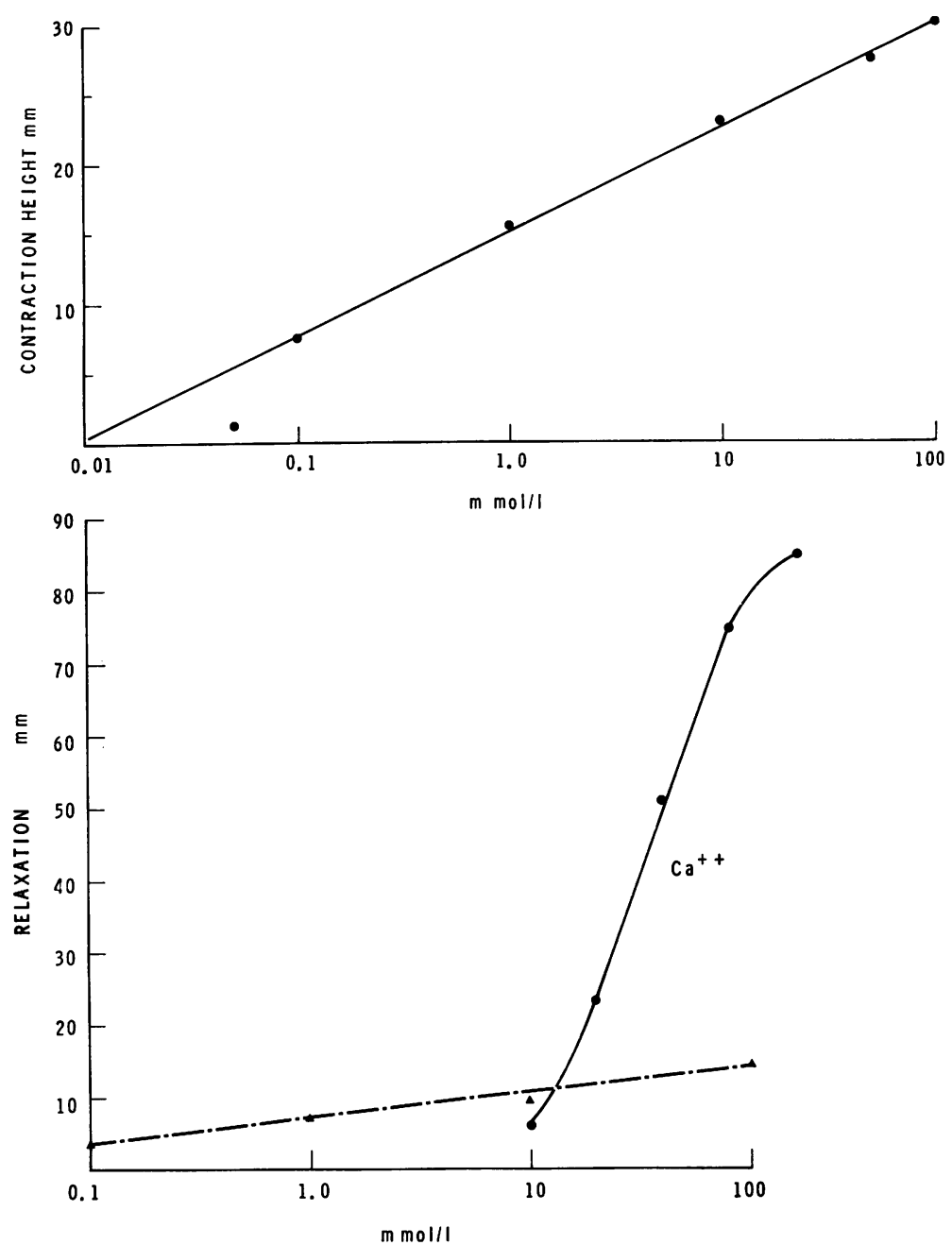

FIG. 5 Effects of cations upon the basilar artery. Upper record, dose/response relationships for relaxation of basilar arteries by calcium (continuous line) and magnesium (interrupted line). Lower record, dose/response relationship for contraction of basilar arteries by potassium. The results are presented in the form described in Fig. 2; each point represents the mean response obtained with two different basilar arteries. The ions were added to the organ bath in the form of a chloride salt for calcium and potassium and the sulphate septahydrate for magnesium.

only of small magnitude. Addition of CSF from both NVS and VS patient groups did not increase the cation concentration in the organ bath by more than $0.5 \mu \mathrm{mol} / 1$.

EFFECTS OF CSF ON DRUG RESPONSES We have previously reported that normal human serum potentiates the contractile responses of the basilar artery to 5-HT and NA (Starling et al., 1975). We therefore investigated the effects of CSF on arterial responses to 5-HT, NA, and the prostaglandins, expecially $\mathrm{PGE}_{2}$. The contractile effects of these compounds are potentiated by CSF from patients with spasm (Fig. 6). In most experiments, 


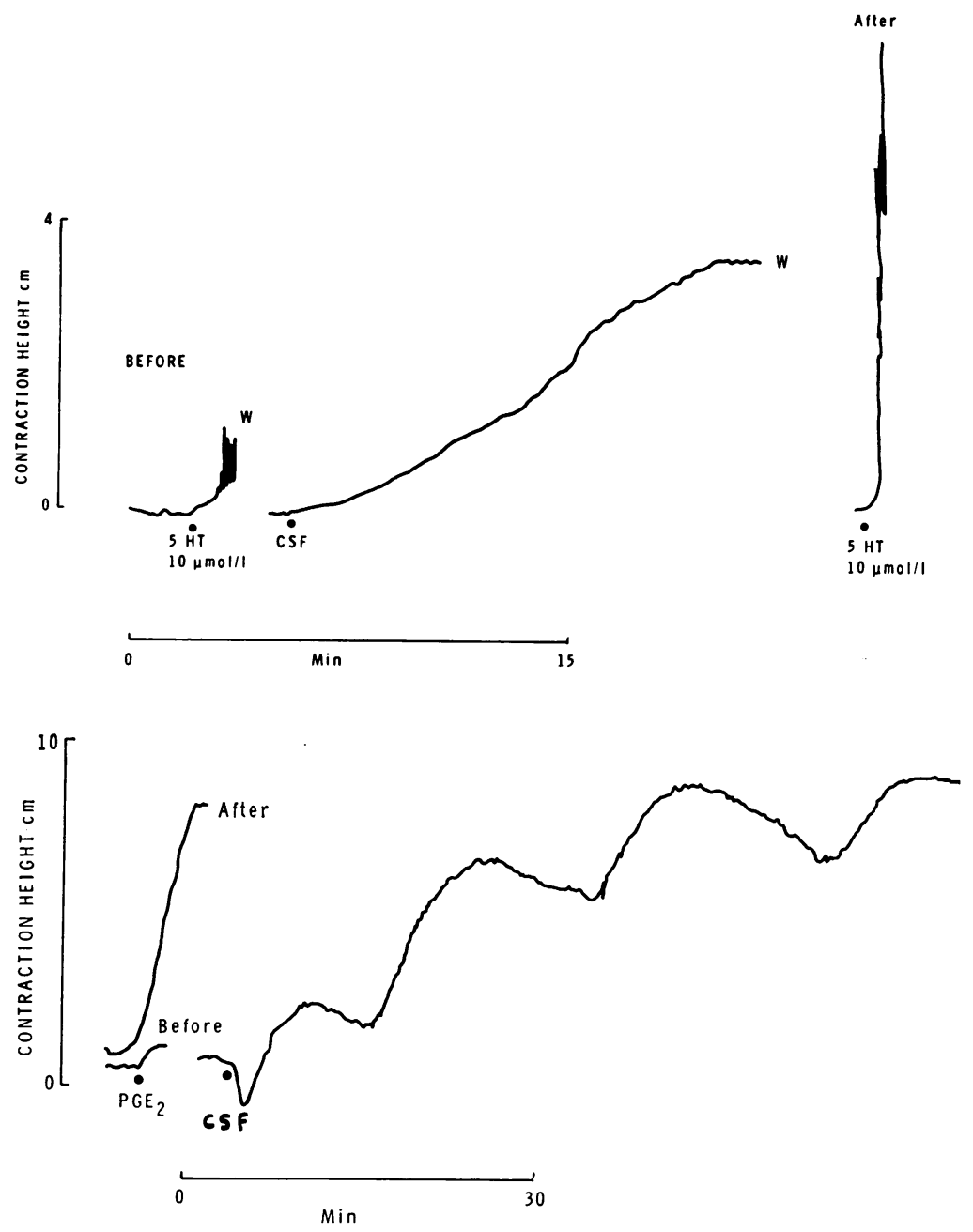

FIG. 6 Potentiation of basilar artery contractions to 5-HT and PGE caused by CSF from patients with subarachnoid haemorrhage and arterial spasm. Records presented in the form shown in Fig. 2 with the time scales (abscissae) only applicable during contractions. In each experiment the artery was first tested to 5-HT or PGE 2 and then the artery was washed and rested for 10-30 min until the arterial length declined to the pre-drug value. Then lyophilized CSF was applied and allowed to remain in contact with the artery for the time indicated. Thereafter the washing and resting periods were repeated and the initial drug retested in the same concentration. Upper record: responses to 5-HT before and after CSF. Lower record: similar responses to PGE $E_{2}$. Note that the PGE response after CSF is placed above the record obtained before CSF. Each record involved a separate experiment with CSF from different patients. 
the contractility of arteries was determined by initial doses of 5-HT, NA, or $\mathrm{PGE}_{2}$. Thereafter the arteries were washed and CSF applied for 0.5 -six hours (normally 0.5 to two hours). At the end of this time the arteries were washed again and then the response to the original drug retested. In a total of 44 tests with 20 subjects $5-\mathrm{HT}$ and $\mathrm{PGE}_{2}$ responses were invariably increased 2.5 to 10 times. We have also observed similar potentiation of the responses to all the other prostaglandins. The effects on NA were variable. In some instances there was two-fourfold potentiation and in others no change in response.

Two types of control experiment were made for comparison. In the first series we investigated the effects of CSF taken from patients with subarachnoid haemorrhage, but without preoperative arterial spasm, upon responses to 5-HT and the prostaglandins. In 23 tests with CSF from nine subiects, the responses to $5-\mathrm{HT}, \mathrm{PGE}_{1}$, $\mathrm{PGE}_{2}, \mathrm{PGA}_{1}, \mathrm{PGA}_{2}$, and $\mathrm{PGF}_{2 \alpha}$ were not increased by more than 1.7 times the initial response. Similar results were obtained in the second type of control experiment involving immersion of the basilar artery in Krebs solution alone for 0.5 to two hours between drug applications.

ANTAGONISM OF CONTRACTIONS INDUCED WITH CSF There were two reasons why it was important to see if the contractions produced by CSF from the patients with subarachnoid haemorrhage could be reversed by means of pharmacological antagonists-firstly, because pharmacological antagonism might aid in the identification of the vasoconstrictor substances present in the CSF, and, secondly, because of the therapeutic implications of being able to reverse the spasm in an in vitro model system.

The following substances have been tested and have failed to modify CSF contractile responses: 2-bromolysergic acid diethylamide and methysergide (5-HT antagonists); phentolamine (a-blocker), mepyramine (antihistamine); and atropine(anticholinergic). These drugs were tested in concentrations of up to $10 \mu \mathrm{mol} / \mathrm{l}$ without affecting CSF responses (Fig. 3). We have found that these concentrations completely abolish responses of the basilar artery to 5-HT, NA, and histamine in concentrations up to $10 \mu \mathrm{mol} / 1$ (Starling et al., 1975).

\section{DISCUSSION}

The cause of cerebral arterial spasm after subarachnoid haemorrhage remains unknown. Most workers agree that it is multifactorial, involving unidentified factors in addition to 5-HT, prostaglandins, and possibly catecholamines (Karlsberg et al., 1963; Kapp et al., 1968a, b; Echlin, $1968,1971)$. As the isolated human basilar artery is contracted by these agents we consider that it is a suitable model for investigating the aetiology of cerebral arterial spasm.

To our knowledge this is the first investigation to show that CSF taken preoperatively from patients with subarachnoid haemorrhage and spasm contains an unidentified substance or substances that contract the isolated human basilar artery, although similar experiments have been made with animal arteries (Buckell, 1964; Wilkins et al., 1967; Kapp et al., 1968a, b; Allen et al., 1974a, b).

Previously we described the pharmacological response of this artery to 5-HT and NA and showed that human serum potentiated these contractile responses (Starling et al., 1975). This finding was important as the presence of blood in the subarachnoid space is an essential feature of cerebral arterial spasm (Kapp et al., 1968a, b; Echlin, 1971). Apart from the observation of the contractility of the isolated artery to CSF from patients with spasm, we have now obtained additional information regarding the basic pharmacological responses of the artery. These are relevant to the cause of spasm. We confirm the work of Miller et al. (1971) that the isolated artery displays spontaneous and rhythmic activity. The involvement of neurohumoral transmitters in this activity is unknown. We have now shown that the human basilar artery is especially sensitive to 5-HT and to six prostaglandins and considerably less sensitive to NA in agreement with work on animal cerebral arteries (for references see Starling et al., 1975). The potency of the prostaglandins has not been investigated in detail, but they appear to have similar potency and contract the artery in low concentrations (up to $200 \mathrm{pmol} / \mathrm{ml}$ ). Although these are considerably less than the concentrations (up to $3 \mathrm{pmol} / \mathrm{ml}$ ) reported by La Torre et al. (1974) in the CSF of patients with subarachnoid haemorrhage from ruptured aneurysms, Pennink et al. (1972) showed that intracisternal injection of 
$20 \mu \mathrm{g} / \mathrm{kg} \mathrm{PGF}_{2 \alpha}$ could produce cerebral arterial spasm in dogs.

White et al. (1975) made separate intracisternal injections of platelets, platelet extracts, whole blood and various other blood components, 5 -HT, histamine, and several prostaglandins and found that all these agents produced some degree of spasm. Also, Holmes (1970) found that infusion of considerable amounts of 5-HT ( $20 \mu \mathrm{g}$ base) into the cerebral ventricles of dogs produced a fourfold increase in release of $E$ series prostaglandins. As this effect was specific to 5 -HT, not being produced by NA or adrenaline, he speculated that the interactions between 5-HT and cerebral prostaglandins warranted further investigation.

There is much other work to suggest that prostaglandins do play some role in cerebral arterial spasm (Denton et al., 1972; Pelofsky et al., 1972; Yamamoto et al., 1972; Nakano et al., 1973).

However, our own observations do not indicate that any of the prostaglandins we have investigated play a major causative role in spasm. This is because the time course of prostaglandin contractions is very rapid, and in contrast with the slowly developing contractions produced by CSF taken from patients with spasm.

The evidence against 5-HT and NA is even stronger. Not only are these contractions rapid, like the prostaglandins, but also they are completely antagonized by specific pharmacological antagonists (Starling et al., 1975). Additionally, these antagonists do not block CSF-induced contractions.

On the other hand, involvement of the prostaglandins and 5-HT cannot be eliminated. This follows from our finding that CSF from patients with spasm potentiates the responses to $5-\mathrm{HT}$, prostaglandins, and to a lesser extent to catecholamines, while CSF from patients without spasm rarely shows such potentiation. At the present state of our investigations we believe that the finding of potentiation is important in the aetiology of spasm. Previously, Raynor et al. (1961) reported potentiation of 5-HT-induced contractions of cat cerebral arteries by CSF from a patient with subarachnoid haemorrhage. Zervas et al. (1973) found that platelet 5-HT potentiated basilar artery spasm in the dog and Bevan et al. (1975) described histamine-induced potentiation of 5-HT and NA contractions.
There is already considerable experimental evidence implicating 5-HT in early and later spasm (Allen et al., 1974b; Zervas et al., 1974). Accordingly, prolonged spasm may be due to a combination of factors: (1) sustained synthesis and release of 5-HT and one or more prostaglandins; (2) a potentiation of these effects by the unidentified contractile substance in CSF from patients with spasm; and (3) the direct contracting effect of this substance itself. These data confirm the generally accepted view that cerebral arterial spasm is caused by the action of pharmacologically active substances on cerebral arteries (Echlin, 1968, 1971; Kapp et al., 1968a, b). Our data show that the changes in the extracellular concentration of $\mathrm{Ca}^{++}, \mathrm{Mg}^{++}$, or $\mathrm{K}^{+}$were not responsible for the changes in arterial tone. $\mathrm{K}^{+}$ contracted and $\mathrm{Ca}^{++}$and $\mathrm{Mg}^{++}$relaxed the artery. They did so only in concentrations well outside the physiological range $\left(\mathrm{Ca}^{++}\right.$and $\left.\mathrm{Mg}^{++}\right)$ or the responses were very small $\left(\mathrm{K}^{+}\right)$.

It was of interest to find that a small number of samples of CSF from patients with and without spasm relaxed the artery, as this had not been observed previously with control CSF (Starling et al., 1975). The time course of these relaxations was short in comparison with the prolonged contractions produced in 16 of the 22 patients with spasm but in only two of the 12 without spasm. There is therefore the possibility of a relaxing factor in addition to the contractile substance.

In conclusion, our results show that CSF taken preoperatively from patients with subarachnoid haemorrhage and cerebral arterial spasm contains one or more substances which contract the isolated human basilar artery. In a small number of patients there is also a relaxing factor, but the distribution of this factor is not related to the presence of spasm.

Our data suggest that the unidentified contracting factor may play a major role in the production of prolonged spasm and that 5-HT, prostaglandins, and possibly NA may be involved in a secondary capacity, because their actions are potentiated by CSF containing the contractile substances. Therefore prolonged arterial spasm appears to involve interaction between the above substances and the unidentified agent. The source of this factor is not known but it is probably derived from blood. 
We would like to thank $\mathrm{Mr} \mathrm{C}$. B. T. Adams, $\mathrm{Mr} \mathrm{M}$. Briggs, and Mr R. S. Gye for allowing us to study these patients and Mrs L. M. Starling for technical assistance.

\section{REFERENCES}

Allen, G. S., Henderson, L. M., Chou, S. N., and French, L. A. (1974a). Cerebral arterial spasm part 1: in vitro contractile activity of vasoactive agents on canine basilar and middle cerebral arteries. Journal of Neurosurgery, 40, 433-441.

Allen, G. S., Henderson, L. M., Chou, S. N., and French, L. A. (1974b). Cerebral arterial spasm part 2: in vitro contractile activity of serotonin in human serum and CSF on the canine basilar artery, and its blockage by methysergide and phenoxybenzamine. Journal of Neurosurgery, 40, 442-450.

Bevan, J. A., Duckles, S. P., and Lee, T. J-F. (1975) Histamine potentiation of nerve and drug-induced responses of a rabbit cerebral artery. Circulation Research, 36, 647-653.

Boullin, D. J., Grahame-Smith, D. G., Mohan, J., and Starling, L. M. (1975a). Contractile activity of cerebrospinal fluid (CSF) from patients with subarachnoid haemorrhage upon the isolated basilar artery. Clinical Science and Molecular Medicine, 49, 6P.

Boullin, D. J., Starling, L. M., Grahame-Smith, D. G.' and Mohan, J. (1975b). Contractile responses of isolated human basilar arteries to cerebrospinal fluid from patients with subarachnoid haemorrhage and arterial spasm. Fifth Congress of the European Association of Neurosurgical Societies, Oxford 1975, pp. 164-166. Department of Neurosurgical Science, Radcliffe Infirmary: Oxford.

Buckell, M. (1964). Demonstration of substances capable of contracting smooth muscle in the haematoma fluid from certain cases of ruptured cerebral aneurysm. Journal of Neurology, Neurosurgery, and Psychiatry, 27, 198-199.

Denton, I. C., White, R. P., and Robertson, J. T. (1972). The effects of prostaglandins $E_{1}, A_{1}$ and $F_{2}$, on the cerebral circulation of dogs and monkeys. Journal of Neurosurgery, 36, 34-42.

Echlin, F. A. (1968). Current concepts in the etiology and treatment of vasospasm. Clinical Neurosurgery, 15, 133-160.

Echlin, F. A. (1971). Experimental vasospasm, acute and chronic, due to blood in the subarachnoid space. Journal of Neurosurgery, 35, 646-655.

Holmes, S. W. (1970). The spontaneous release of prostaglandins into the cerebral ventricles of the dog and the effect of external factors on this release. British Journal of Pharmacology, 38, 653-658.
Kapp, J., Mahaley, M. S., and Odom, G. L. (1968a). Cerebral arterial spasm part 2: Experimental evaluation of mechanical and humoral factors in pathogenesis. Journal of Neurosurgery, 29, 339-349.

Kapp, J., Mahaley, M. S., and Odom, G. L. (1968b). Cerebral arterial spasm part 3: Partial purification and characterization of a spasmogenic substance in feline platelets. Journal of Neurosurgery, 29, 350-355.

Karlsberg, P., Elliott, H. W., and Adams, J. E. (1963). Effect of various pharmacologic agents on cerebral arteries. Neurology (Minneap.), 13, 772-778.

La Torre, E., Patrono, C., Fortuna, A., and GrossiBelloni, D. (1974). Role of prostaglandin $F_{2 a}$ in human cerebral vasospasm. Journal of Neurosurgery, 41, 293-299.

Miller, C. A., Yashon, D., Locke, G., and Hunt, W. E. (1971). Autonomous activity in the human basilar artery: Relationship to cerebral vascular spasm. Neurology (Minneap.), 21, 1249-1254.

Nakano, J., Chang, A. C., and Fisher, R. G. (1973). Effects of prostaglandins $E_{1}, E_{2}, A_{1}, A_{2}$, and $F_{2 a}$ on canine carotid arterial blood flow, cerebrospinal fluid pressure and intraocular pressure. Journal of Neurosurgery, 38, 32-39.

Pelofsky, S., Jacobson, E. D., and Fisher, R. G. (1972). Effects of prostaglandin $E_{1}$ on experimental cerebral vasospasm. Journal of Neurosurgery, 36, 634-639.

Pennink, M., White, R. P., Crockarell, J. R., and Robertson, J. T. (1972). Role of prostaglandin $F_{2 a}$ in the genesis of experimental cerebral vasospasm: Angiographic study in dogs. Journal of Neurosurgery, 37, 398-406.

Raynor, R. B., McMurtry, J. G., and Pool, J. L. (1961.) Cerebrovascular effects of topically applied serotonin in the cat. Neurology (Minneap.), 11, 190-195.

Starling, L. M., Boullin, D. J., Grahame-Smith, D. G., Adams, C. B. T., and Gye, R. S. (1975). Responses of isolated human basilar arteries to 5-hydroxytryptamine, noradrenaline, serum, platelets and erythrocytes. Journal of Neurology, Neurosurgery, and Psychiatry, 38, 650-656.

White, R. P., Hagen, A. A., Morgan, H., Davison, W. N., and Robertson, J. T. (1975). Experimental study on the genesis of cerebral vasospasm. Stroke, 6, 52-57.

Wilkins, R. H., Wilkins, G. K., Gunnells, J. C., and Odom, G. L. (1967). Experimental studies of intracranial arterial spasm using aortic strip assays. Journal of Neurosurgery, 27, 490-500.

Yamamoto, Y. L., Feindel, W., Wolfe, L. S., Katoh, H., and Hodge, C. P. (1972). Experimental vasoconstriction of cerebral arteries by prostaglandins. Journal of Neurosurgery, 37, 385-397.

Zervas, N. T., Kuwayama, A., Rosoff, C. B., and Salzman, E. W. (1973). Cerebral arterial spasm: modification by inhibition of platelet function. Archives of Neurology (Chic.), 28, 400-404. 